\title{
New Application Approval
}

\section{Abiraterone acetate}

Approved date: July 20, 2017

Treatment for: Prostate Cancer

Abiraterone acetate is an improved ultramicrosize formulation of the oral CYP17 inhibitor abiraterone acetate (approved as Zytiga) in development for the combination treatment of metastatic castration-resistant prostate cancer.

\section{Buprenorphine}

Approved date: July 20, 2017

Treatment for: Opiate Dependence

Buprenorphine is a long-acting partial opioid agonist formulation in development for the treatment of opioid dependence.

\section{Erenumab}

Approved date: July 20, 2017

Treatment for: Migraine Prophylaxis

Erenumab is a human monoclonal antibody that inhibits the receptor for calcitonin gene-related peptide (CGRP) in development for the prevention of episodic and chronic migraine.

\section{Romosozumab}

Approved date: July 16, 2017

Treatment for: Osteoporosis

Romosozumab is an anti-sclerostin monoclonal antibody in development for the treatment of osteoporosis in postmenopausal women at increased risk of fracture.

\section{$\underline{\text { Abemaciclib }}$}

Approved date: July 12, 2017

Treatment for: Breast Cancer

Abemaciclib is a selective ATP-competitive inhibitor of cyclin dependent kinases (CDK) 4 and 6 in development for the treatment of metastatic breast cancer.

\section{Migalastat}

Approved date: July 11, 2017

Treatment for: Fabry Disease

Migalastat is an investigational pharmacological

chaperone in development for the treatment of Fabry

disease.

\section{Dexamethasone (Intracanalicular Depot)}

Approved date: July 11, 2017

Treatment for: Postoperative Ocular Inflammation

Dexamethasone is an investigational intracanalicular depot corticosteroid formulation designed to deliver sustained release dexamethasone to the ocular surface for up to 30 days after ophthalmic surgery for the treatment of post-surgical ocular inflammation and pain.

\section{Entrectinib}

Approved date: July 10, 2017

Treatment for: Neuroblastoma

Entrectinib is a potent, novel, orally available, selective tyrosine kinase inhibitor in development for the treatment of neuroblastoma.

\section{Cyclobenzaprine hydrochloride}

Approved date: July 10, 2017

Treatment for: Post Traumatic Stress Disorder

Cyclobenzaprine is a sublingual formulation of an approved skeletal muscle relaxant in development for the treatment of patients with post-traumatic stress disorder (PTSD).

\section{Treprostinil}

Approved date: June 30, 2017

Treatment for: Pulmonary Arterial Hypertension

Treprostinil is a preservative-free, parenteral formulation of the approved vasodilatory prostacyclin analogue treprostinil delivered via the proprietary 
PatchPump infusion system for the treatment of pulmonary arterial hypertension (PAH).

\section{Levodopa (Inhalation Powder)}

Approved date: June 29, 2017

Treatment for: Parkinson's disease

Levodopa is an oral inhalation formulation of the approved drug levodopa in development as a treatment for symptoms of OFF periods in people with Parkinson's disease taking a carbidopa/levodopa regimen.

\section{Fostamatinib}

Approved date: June 19, 2017

Treatment for: Immune Thrombocytopenia

Fostamatinib is an investigational oral spleen tyrosine kinase (SYK) inhibitor in development for the treatment of patients with chronic and persistent immune thrombocytopenia (ITP).

\section{Bictegravir, Emtricitabine \& Tenofovir Alafenamide}

Approved date: June 12, 2017

Treatment for: HIV Infection

Bictegravir, emtricitabine and tenofovir alafenamide is an investigational integrase strand transfer inhibitor and emtricitabine/tenofovir alafenamide (FTC/TAF) combination in development for the treatment of HIV-1 infection in adults.

Source: drugs.com

Information collected and compiled by:

Mr. Md. Akbar Hossain

Assistant Professor

Department of Pharmacy

ASA University, Dhaka 